\title{
Pembrolizumab in the treatment of metastatic non-small-cell lung cancer: patient selection and perspectives
}

This article was published in the following Dove Press journal:

Lung Cancer:Targets and Therapy

II January 2017

Number of times this article has been viewed

\author{
Ashwin Somasundaram \\ Timothy F Burns \\ Division of Hematology/Oncology, \\ Department of Medicine, University \\ of Pittsburgh Cancer Institute, \\ Pittsburgh, PA, USA
}

\begin{abstract}
Lung cancer is the leading killer of both men and women in the US, and the 5-year survival remains poor. However, the approval of checkpoint blockade immunotherapy has shifted the treatment paradigm and provides hope for improved survival. The ability of nonsmall-cell lung cancer (NSCLC) to evade the host immune system can be overcome by agents such as pembrolizumab (MK-3475/lambrolizumab), which is a monoclonal antibody targeting the programmed death 1 (PD-1) receptor. In early studies, treatment with pembrolizumab led to dramatic and durable responses in select patients (PD-L1+ tumors). This remarkable efficacy lead to approval of pembrolizumab in the second-line setting as response rates were almost doubled compared to standard of care (SOC) chemotherapy. Most recently, data in the first-line setting from the KEYNOTE-024 study have redefined the SOC therapy for a selected subset of patients. In patients with $\geq 50 \%$ PD-L1+ tumors, pembrolizumab had a clear progressionfree survival and overall survival benefit. Toxicity was mostly immune related and similar to checkpoint blockade toxicities observed in previous studies. The initial approval and subsequent studies of pembrolizumab required and utilized a companion diagnostic test, Dako's IHC 22C3, to assess PD-L1 status of patients. The evaluation and scoring system of this assay has been used by other companies as a reference to develop their own assays, which may complicate selection of patients. Finally, the impact of pembrolizumab in NSCLC is growing as evidenced by the numerous, ongoing trials open for combinations with chemotherapy, chemoradiation, other immunotherapeutics, immunomodulators, tyrosine kinase inhibitors, PI3K inhibitors, MEK inhibitors, hypomethylating agents, and histone deacetylase inhibitors. Further studies are also evaluating pembrolizumab in small-cell lung cancer and malignant pleural mesothelioma. This explosion of studies truly conveys the lack of therapeutic answers for lung cancer patients and the promise of pembrolizumab.
\end{abstract}

Keywords: NSCLC, programmed death 1, programmed death ligand 1, tumor-infiltrating lymphocytes, regulatory T-cells

\section{Introduction}

Lung cancer is a devastating disease with more than 224,390 new cases and more than 158,080 deaths estimated for 2016 in the US alone. The overall survival (OS) of lung cancer remains low, but the recent successes of immunotherapy and checkpoint blockade show the promise to improve long-term survival. ${ }^{1}$ A "hallmark of cancer" is the ability of the tumor to evade the immune system. ${ }^{2}$ The body's adaptive immune system fights cancer cells through the activation of T-cells and is regulated through various checkpoint signals. Antigen-presenting cells (APCs) recognize cancer cells 
as foreign and present cancer antigens to lymphocytes to activate them and cause proliferation. These tumor infiltrating lymphocytes (TILs) include CD4+ and CD8+T-cells and aid in destruction of the tumor. The APCs and regulatory T-cells (Tregs) prevent a positive feedback loop of T-cell activation through checkpoint signals. These signals halt excessive T-cell activation and proliferation and prevent autoimmunity. Unfortunately, tumors also utilize and, in some cases, are dependent upon these signals to escape immune-mediated destruction. Blockade of these checkpoint signals, such as programmed death L1 (PD-L1)/PD-1, can reactivate an immune response against a tumor (Figure 1). Historically, lung cancer was not thought to be immunogenic; however, the observation that these checkpoint signals were elevated in non-small-cell lung cancer (NSCLC) suggested that it may be immunogenic. Among the checkpoint signals that were increased were the cytotoxic $\mathrm{T}$ lymphocyte-associated molecule-4 (CTLA-4) and PD-L1. PD-L1 can be found on APCs and Tregs and can bind to PD-1 to attenuate the T-cell response during inflammation. However, cancer cells can also express PD-L1 to mimic this interaction, leading to T-cell suppression. ${ }^{3,4}$

The first checkpoint blockade agents to gain approval in oncology were those agents that blocked CTLA-4. These agents demonstrated significant efficacy in melanoma but had only modest activity in NSCLC. ${ }^{5}$ Alternatively, increased levels of PD-L1 correlated with the number of TILs in NSCLC and suggested that PD-1 inhibition would be a promising target. ${ }^{6}$ Early studies with the PD-1 inhibitors included the monoclonal antibodies nivolumab and pembrolizumab, which showed great efficacy in melanoma. ${ }^{7}$ Similar findings were seen in NSCLC with nivolumab, which led to its approval in the pretreated setting for both squamous and nonsquamous patients. ${ }^{89}$ Subsequently, pembrolizumab was approved as well for use in NSCLC after demonstrating improved OS and more durable responses compared to standard of care (SOC) second-line docetaxel. ${ }^{10}$ Unfortunately, most patients with lung cancer still do not respond to pembrolizumab monotherapy, and further study is warranted.

\section{Monotherapy in the second-line setting and beyond}

The first study evaluating pembrolizumab in NSCLC was KEYNOTE-001. It was an early Phase I/II, biomarker-driven study demonstrating safety and efficacy of pembrolizumab in patients with PD-L1-positive advanced NSCLC. PD-L1 was assessed by Dako's companion diagnostic IHC 22C3 in KEYNOTE-001 and defined as at least 1\% staining of the cells lining the tumor tissue (Table 1). ${ }^{10}$ Based on the early success of KEYNOTE-001, pembrolizumab was further evaluated in NSCLC to determine the relationship between dose and response in KEYNOTE-025. Pembrolizumab $2 \mathrm{mg} /$ $\mathrm{kg}$ q3 weeks was compared to pembrolizumab $10 \mathrm{mg} / \mathrm{kg}$ q3 weeks and pembrolizumab $10 \mathrm{mg} / \mathrm{kg}$ q2 weeks with radiographic assessment every 9 weeks. Overall response rate (ORR) was 15\% (95\% CI 7-28) with pembrolizumab

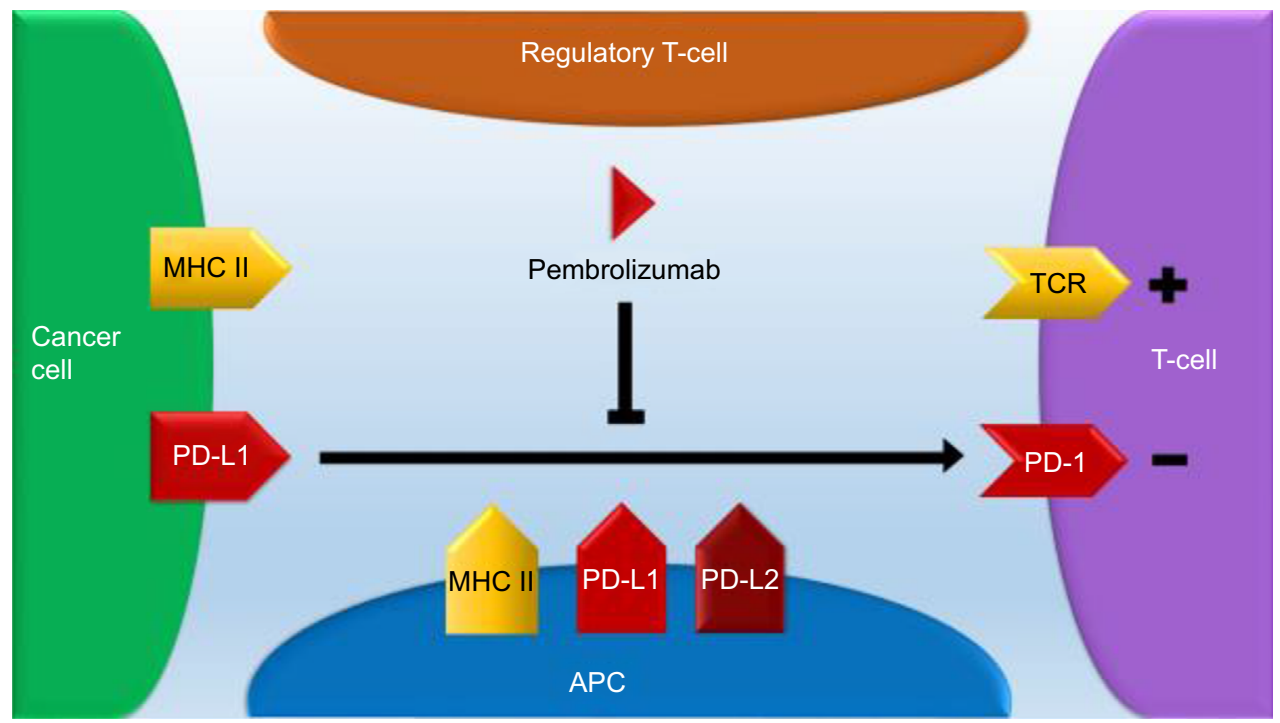

Figure I Pembrolizumab blocks a critical inhibitory signal from tumor cells allowing T-cell activation and antitumor immune response.

Notes: T-cells interact with APCs through the T-cell receptor binding to the APC MHC surface molecule-presenting antigen. Afterward, T-cell proliferation and activation occur against the presented antigen. However, ligation of PD-I on T-cells to PD-LI present on APCs, tumor cells, or Tregs can dampen this response. Pembrolizumab inhibits PD-I binding to PD-LI allowing T-cell activation and an antitumor immune response. In addition, pembrolizumab also prevents the interaction between PD-I and PD-L2, which may or may not be beneficial for an immune tumor response.

Abbreviations: APCs, antigen-presenting cells; PD-I, programmed death I; Tregs, regulatory T-cells; TCR, T-cell receptor; MHC, major histocompatibility complex; PD-LI, programmed death-ligand I; PD-L2, programmed death-ligand 2. 
Table I Monotherapy in the second-line setting and beyond

\begin{tabular}{lllllll}
\hline Trial name & Phase & Histology & Therapy & mPFS & I-Year PFS & mORR \\
\hline KEYNOTE-00I & I/II & All & PEMBRO 2Q3W, I0Q3W, I0Q2W & NR & NR & Ref \\
KEYNOTE-0I0 & II/III & All & PEMBRO 2 (PD-LI+) & 3.9 months & NR & NR \\
& & & & mOS & I-Year OS & DOR \\
& & & PEMBRO 2Q3W, I0Q3W, I0Q2W & NR & NR & NR \\
& & & PEMBRO 2 (PD-LI+) & 9.4 months (14.9) & NR & NR \\
\hline
\end{tabular}

Note: Completed trials in NSCLC.

Abbreviations: mPFS, median progression-free survival; PEMBRO, pembrolizumab; NR, not reported; PD-LI, programmed death LI; mOS, median overall survival; NSCLC, non-small-cell lung cancer; mORR, median overall response rate; DOR, duration of response.

$2 \mathrm{mg} / \mathrm{kg}$ and $25 \%(95 \% \mathrm{CI} 18-33)$ with pembrolizumab $10 \mathrm{mg} / \mathrm{kg}$ and $21 \%$ (95\% CI 14-30) with pembrolizumab $10 \mathrm{mg} / \mathrm{kg}$ q2 weeks. A regression analysis between area under the curve (AUC) dose and response, along with adverse events (AEs), found no significant exposure dependence for efficacy or safety in patients. AEs included fatigue (14\%), dermatitis $(6 \%)$, diarrhea $(6 \%)$, pruritus $(8 \%)$, or dyspnea $(6 \%)$ and a single serious drug-related AE (grade 3 pericardial effusion). ${ }^{11}$ KEYNOTE-025 has completed accrual, but follow-up is ongoing. ${ }^{12}$

Based on the promising data of KEYNOTE-001, further studies were conducted in the Phase II/III settings. KEYNOTE-010 was a Phase II/III, biomarker-driven study that evaluated pembrolizumab versus SOC docetaxel in patients with PD-L1-positive ( $>1 \%)$, pretreated, advanced NSCLC. The median OS was 8.5 months versus 10.4 months versus 12.7 months for docetaxel, pembrolizumab $2 \mathrm{mg} / \mathrm{kg}$, and pembrolizumab $10 \mathrm{mg} / \mathrm{kg}$, respectively (Table 1). Both pembrolizumab $2 \mathrm{mg} / \mathrm{kg}$ (hazard ratio [HR] 0.71, 95\% CI $0.58-0.88 ; p=0.0008)$ and pembrolizumab $10 \mathrm{mg} / \mathrm{kg}(\mathrm{HR}$ $0.61,95 \%$ CI $0.49-0.75 ; p<0.0001)$ demonstrated a significant OS benefit when compared to docetaxel. In the subgroup of patients with $\geq 50 \%$ PD-L1 tumors, the OS advantage for pembrolizumab $2 \mathrm{mg} / \mathrm{kg}$ or pembrolizumab $10 \mathrm{mg} / \mathrm{kg}$ versus docetaxel was even greater (14.9 months versus 8.2 months; HR 0.54 , 95\% CI $0.38-0.77 ; p=0.0002$ and 17.3 months versus 8.2 months; HR $0.50,95 \%$ CI $0.36-0.70$; $p<0.0001$ ). The median progression-free survival (PFS) was 4.0 months versus 3.9 months versus 4.0 months for docetaxel, pembrolizumab $2 \mathrm{mg} / \mathrm{kg}$, and pembrolizumab $10 \mathrm{mg} / \mathrm{kg}$, respectively. Conversely, in the subgroup of patients with $\geq 50 \%$ PD-L1 tumors, pembrolizumab $2 \mathrm{mg} / \mathrm{kg}$ treatment led to a longer PFS compared to docetaxel (5.0 months versus 4.1 months; HR 0.59, 95\% CI 0.44-0.78; $p=0.0001)$. Similar results were observed with pembrolizumab $10 \mathrm{mg} / \mathrm{kg}$ compared to docetaxel in this subgroup (5.2 months versus 4.1 months; HR $0.59,95 \%$ CI $0.45-0.78 ; p<0.0001)$. This study suggested the importance of biomarker use in pembrolizumab therapy for patient selection. Treatment-related adverse events (TrAEs) were also less frequent with pembrolizumab $2 \mathrm{mg} / \mathrm{kg}$ and pembrolizumab $10 \mathrm{mg} / \mathrm{kg}$ ( $13 \%$ and $16 \%$ respectively) compared to docetaxel (35\%), suggesting pembrolizumab is also more tolerable than SOC therapy. AEs occurred at a rate of $20 \%$ in the pembrolizumab $2 \mathrm{mg} / \mathrm{kg}$ arm and $19 \%$ in the pembrolizumab $10 \mathrm{mg} / \mathrm{kg}$ arm. The most common AEs included colitis (1\%), hyper- and hypothyroidism (12\%), severe skin reactions (grades 3 to 5 at 1\%), and pneumonitis (grades 3 to 5 at $2 \%$ ). In this study, PD-L1 was again evaluated with the Dako's companion diagnostic, and based on these remarkable findings, pembrolizumab, and its diagnostic test, received expedited US Food and Drug Administration approval. ${ }^{13}$

Of note, the 1034 patients enrolled in KEYNOTE-010 had their tumor samples reassessed with both archival (456 samples or $44 \%$ ) and new samples (578 samples or $56 \%$ ). There was high correlation in staining between archival versus new samples as $40 \%$ of the archival samples displayed $\geq 50 \%$ PD-L1 expression versus $45 \%$ of new samples. Furthermore, regardless of archival or new sampling, PD-L1 high or low status, pembrolizumab demonstrated significant OS benefit compared to docetaxel. With similar PD-L1 expression and outcome regardless of archival or new sampling, this retrospective data suggested that new sampling to assess PD-L1 status may be unnecessary. ${ }^{13}$

\section{Monotherapy in the first-line setting}

Although anti-PD-1 inhibitors were found to be superior to chemotherapy in the second-line setting, it was unknown whether prior chemotherapy was required for the efficacy of these agents or whether they would be superior to first-line cytotoxic therapy where the established response rates and OS benefits are significantly greater than the second-line setting. This question was addressed with pembrolizumab in the Phase III setting in the recently reported KEYNOTE-024 study (Table 2). ${ }^{14}$ Patients with advanced, $\geq 50 \%$ PD-L1-positive, treatment-naive NSCLC were randomized to first-line pembrolizumab $200 \mathrm{mg} \mathrm{q3}$ weeks versus SOC 
Table 2 Monotherapy in the first-line setting

\begin{tabular}{|c|c|c|c|c|c|c|c|}
\hline Trial name & Phase & Histology & Therapy & mPFS & 6-month PFS & mORR & Ref \\
\hline \multirow[t]{3}{*}{ KEYNOTE-024 } & III & All & PEMBRO Q3W (PD-LI >50\%) vs PT-DC & 10.3 months vs 6.0 months & $62.1 \%$ vs $50.3 \%$ & $44.8 \%$ vs $27.8 \%$ & 14 \\
\hline & & & & mos & 6-Month OS & DOR & \\
\hline & & & PEMBRO Q3W (PD-LI >50\%) vs PT-DC & NR vs NR & $80.2 \%$ vs $72.4 \%$ & NR vs 6.3 & \\
\hline
\end{tabular}

Note: Completed trials in NSCLC.

Abbreviations: mPFS, median progression-free survival; PEMBRO, pembrolizumab; PD-LI, programmed death LI; PT-DC, platinum-doublet chemotherapy; mOS, median overall survival; NR, not reported; NSCLC, non-small-cell lung cancer; DOR, duration of response.

platinum-based chemotherapy. This studied stratified patients by histology, location, and Eastern Cooperative Oncology Group (ECOG) and evaluated PFS as the primary end point and OS, ORR, and safety as secondary end points. The 6-month OS was $80.2 \%$ with pembrolizumab versus $72.4 \%$ with chemotherapy with an HR of 0.60 (95\% CI $0.41-0.89$; $p=0.005)$. In addition, the median PFS was 10.3 months (95\% CI 6.7 to not reported [NR]) with pembrolizumab versus 6.0 months (95\% CI 4.2-6.2) with chemotherapy with an HR of 0.50 ( $95 \%$ CI $0.37-0.68 ; p<0.001$ ). ORR was $44.8 \%$ with pembrolizumab versus $27.8 \%$ with chemotherapy. The median duration of the response was not reached with pembrolizumab (1.9+ to $14.5+$ months) versus 6.3 months (2.1+ to $12.6+$ months) with chemotherapy. TrAEs such as diarrhea (14.3\%), fatigue (10.4\%), and fever (10.4\%) occurred in only $26.6 \%$ of patients treated with pembrolizumab. Grade 3 and greater TrAEs with pembrolizumab included diarrhea $(3.9 \%)$ and pneumonitis (2.6\%). Severe TrAEs such as anemia (44.0\%; grade 3 and greater $19.3 \%$ ), neutropenia (grade 3 and greater 13.3\%), thrombocytopenia (grade 3 and greater $5.3 \%$ ), fatigue (28.7\%), and nausea (43.3\%) occurred in $53.3 \%$ of patients treated with chemotherapy. ${ }^{14}$ This study has established a new SOC for the first-line setting for $\sim 30 \%$ of patients with PD-L1-positive ( $>50 \%)$ tumors. Ongoing efforts are underway to improve response rates for the majority of patients who did not respond and determine whether the $70 \%$ of patients with $<50 \%$ PD-L1 positivity may benefit from single-agent pembrolizumab in the first-line setting.

As such, a second international Phase III study, KEYNOTE-042, compares pembrolizumab to platinum-based chemotherapy in patients with advanced, PD-L1+, EGFR/ $A L K$-negative NSCLC in the first-line setting (Table 3). Patients will be randomized to either a flat dose of pembrolizumab $200 \mathrm{mg}$ q3 weeks versus carboplatin plus paclitaxel or carboplatin plus pemetrexed. This study will also compare the subgroup of patients with PD-L1 $\geq 50 \%$ versus PD-L1 1-49\%, based on Dako's 22C3 antibody. Other subgroups will include squamous versus nonsquamous histology, ECOG performance status 0 or 1, and East Asia versus non-East Asia region. Treatment with chemotherapy is planned for six cycles and pembrolizumab is planned until 35 cycles, intolerability, or progression of disease. Pemetrexed maintenance was allowed for both arms and responses will be assessed q9 weeks. The primary end point is OS in PD-L1 $\geq 50 \%$ patients, while secondary end points include PFS in PD-L1 $\geq 50 \%$ patients and OS in all patients..$^{15}$

\section{Combinations with chemotherapy}

In an effort to achieve higher response rates, combinations of SOC cytotoxic chemotherapy with pembrolizumab are being investigated. The Phase I/II study, KEYNOTE-021 is evaluating the efficacy and safety of pembrolizumab in combination with chemotherapy in advanced, EGFR/ALK wild-type NSCLC patients in the first-line setting. Patients will receive carboplatin AUC 6 with pemetrexed or paclitaxel $200 \mathrm{mg} / \mathrm{m}^{2}$ plus bevacizumab for nonsquamous histology and carboplatin AUC 6 with paclitaxel $200 \mathrm{mg} / \mathrm{m}^{2}$ for any histology. These groups will additionally receive either maintenance pembrolizumab $2 \mathrm{mg} / \mathrm{kg}$ or $10 \mathrm{mg} / \mathrm{kg}$ with responses measured in 6-week intervals. Early data show grade 3-4 TrAEs occurring at $36 \%, 46 \%$, and $42 \%$ for patients on maintenance pembrolizumab (cohort A), maintenance pembrolizumab plus bevacizumab (cohort B), and maintenance pembrolizumab and pemetrexed (cohort C), respectively. The most common TrAEs included febrile neutropenia (two in cohorts A and B), neutropenia (two in cohorts A and B), anemia (two in cohorts A and C), AST elevation (three in cohort C), one dose limiting grade 3 rash in cohort $\mathrm{C}$, and one treatment-related death due to pericardial effusion in cohort B. Subgroups were also evaluated based on PD-L1 expression. While safety data are promising, efficacy data are still immature. ${ }^{16}$ Further combinations of chemotherapy and pembrolizumab are being evaluated in the studies KEYNOTE-011 and KEYNOTE-407. KEYNOTE-011 is a Phase I study of pembrolizumab $2 \mathrm{mg} / \mathrm{kg}, 10 \mathrm{mg} / \mathrm{kg}$, and $200 \mathrm{mg}$ monotherapy and pembrolizumab in combination with platinum-based chemotherapy in patients with advanced NSCLC. Chemotherapy combinations with pembrolizumab include cisplatin/ pemetrexed, carboplatin/pemetrexed, carboplatin/paclitaxel, or carboplatin/nab-paclitaxel. This study will evaluate safety 


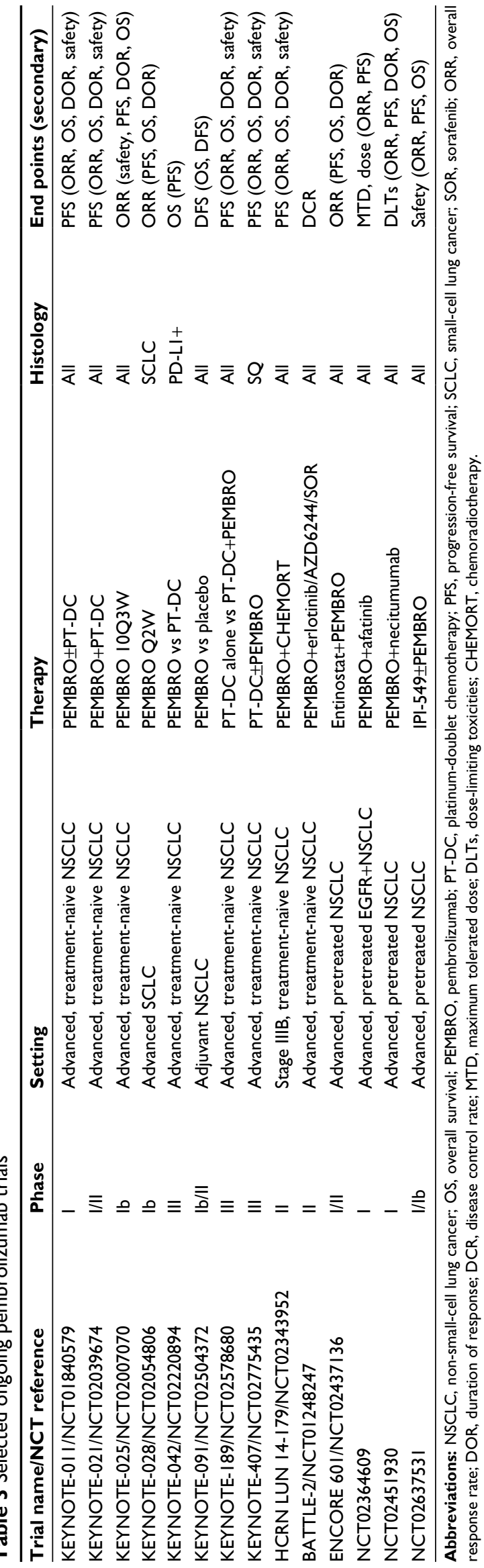

and tolerability of these regimens. ${ }^{17}$ KEYNOTE-407 is also an ongoing, randomized, double-blind, Phase III study, evaluating carboplatin plus paclitaxel/nab-paclitaxel with and without pembrolizumab as first-line therapy in patients with advanced squamous NSCLC. The primary end points will be PFS and OS. ${ }^{18}$ The early findings regarding safety and tolerability from KEYNOTE-021 led to the development of KEYNOTE-189, a Phase III, randomized, double-blind study evaluating the safety and efficacy of first-line platinum-based chemotherapy (pemetrexed $500 \mathrm{mg} / \mathrm{m}^{2}$ with either cisplatin $75 \mathrm{mg} / \mathrm{m}^{2}$ or carboplatin AUC $5 \mathrm{q} 3$ weeks for four cycles) alone versus first-line chemotherapy plus pembrolizumab $200 \mathrm{mg}$ for patients with advanced, nonsquamous, EGFR/ $A L K$-negative NSCLC. Patients will be able to continue on maintenance pemetrexed and placebo or pembrolizumab. Patients will be stratified by PD-L1 status, smoking status, and platinum chemotherapy regimen. The primary end point will be PFS, and the secondary end points will be OS, ORR, duration of response (DOR), and safety. ${ }^{19,20}$

Finally, pembrolizumab is also being evaluated in the combination with SOC chemotherapy in the adjuvant setting. One of the proposed benefits of using immunotherapy in the adjuvant setting is to create a reservoir of memory T-cells that will actively ward-off recurrence of tumor after initial exposure similar to the adaptive immune system's memory after vaccination. However, it is unknown whether the paucity of antigen after resection to elicit a brisk response will prevent the generation of these activated tumor antigen-specific T-cells. There is also the risk of toxicity in a population that may have already been cured by SOC treatment. KEYNOTE-091 also called PEARLS is a Phase III, randomized trial comparing pembrolizumab versus placebo, after SOC adjuvant chemotherapy in patients with resectable, early-stage NSCLC. The study will primarily look at disease-free survival (DFS). ${ }^{21,22}$

\section{Novel combinations}

In order to find effective therapies for those patients who do not benefit from single-agent pembrolizumab, this agent is also being evaluated in combinations with radiotherapy, chemotherapy, targeted therapy, and other immunotherapy agents. Preclinical mouse models have demonstrated a synergistic effect of checkpoint blockade therapy and pembrolizumab after radiation. This synergy has been theorized to be similar to the, "Abscopal phenomenon," where radiation causing DNA damage leads to an increased mutation burden and higher tumor immunogenicity. ${ }^{23,24}$ Therefore, pembrolizumab is being evaluated in patients with unresectable stage III NSCLC in combination with concurrent chemoradiation 
in an ongoing Phase II study, HCRN LUN 14-179. Patients in this study were treated with cisplatin/etoposide, carboplatin/paclitaxel, or cisplatin/pemetrexed with concurrent radiation followed by consolidation chemotherapy and then followed by pembrolizumab $200 \mathrm{mg}$ intravenously q3 weeks after 4-8 weeks, lasting for up to 12 months. The primary end point will be progression of disease outside of the radiation field. Secondary end points will include PFS, OS, and toxicity. This study will also explore the correlation between PD-L1 expression and the study end points. Early data of ten enrolled patients as of January 31, 2016, show one grade 3 urinary toxicity, one grade 1 pneumonitis, and five grade 1-2 complaints of fatigue. ${ }^{24}$

Hypomethylating agents, such as azacitidine, are being evaluated in combination with pembrolizumab in early clinical trials for patients with advanced, pretreated NSCLC. The epigenetic changes from hypomethylating agents have been theorized to increase tumor immune response. An early study evaluating this combination will look at PFS as a primary end point. ORR, disease control rate (DCR), OS, pharmacokinetics (PK), and safety will be secondary end points, while exploratory end points will include DNA methylation, PD-L1 expression, gene expression, and TILs. ${ }^{25}$ Pembrolizumab is also being evaluated in combination with a histone deacetylase inhibitor, entinostat in advanced, pretreated NSCLC in the Phase Ib/II ENCORE 601 study. Early reports suggest that this combination may be tolerable with one grade 3 hepatitis, however, only stable disease was observed in three out of six patients. ${ }^{26,27}$ Both entinostat and azacitidine have been evaluated in combination in pretreated patients, with four out of 19 patients responding and a median survival of 6.4 months, suggesting existing efficacy of these agents. ${ }^{28}$ Furthermore, several of these patients, who subsequently received anti-PD-1 therapy, had dramatic responses, suggesting a possible role for epigenetic priming. Preclinical studies suggest that histone deacetylation can decrease the activity of Tregs, leading to an increased immune response.

$\mathrm{PI} 3 \mathrm{~K}$ is a signaling molecule that is part of many pathways but is prominent in the PI3K/AKT/mTOR pathway, leading to downstream transcription factors necessary for tumor development. Preclinical studies have demonstrated that inhi- bition of PI3K-gamma can lead to increased tumor immune response in the tumor microenvironment and decreased tumor growth. ${ }^{29}$ These findings have led to a Phase Ib study that will be evaluating the PK, maximum tolerated dose (MTD), and safety of IPI-549 (a PI3K-gamma inhibitor) in combination with pembrolizumab in NSCLC.

Pembrolizumab, as well as other PD-1 inhibitors such as nivolumab, is also being explored in combination with the CTLA-4 checkpoint inhibitors, ipilimumab, and with targeted therapies. The ongoing, Phase I/II KEYNOTE-021 study also includes cohorts evaluating pembrolizumab in combination with ipilimumab (CTLA-4 checkpoint inhibitor) for patients with advanced, pretreated NSCLC ${ }^{30}$ (Table 4). In addition, the BATTLE-2 study, is a biomarker, adaptive, multiplatform study utilizing pembrolizumab in combination with targeted agents. This trial will evaluate patients with advanced, pretreated, $E G F R / A L K$-negative NSCLC stratified by $K R A S$ status and assigns patients to erlotinib versus erlotinib plus pembrolizumab, versus pembrolizumab plus selumetinib (AZD6244, an MEK inhibitor), or sorafenib. Assignment into these arms will be based upon response and similar gene expression profiles with next-generation sequencing. It will also explore predictive and prognostic biomarkers and gene profiles. Initial data of 186 patients showed adaptive assignment of 22 patients to erlotinib, 42 patients to erlotinib plus pembrolizumab, 75 patients to pembrolizumab plus selumetinib, and 61 patients to sorafenib with an 8 -week DCR of $32 \%, 50 \%, 53 \%$, and $46 \%$, respectively, and an overall DCR of $48 \%$. KRAS mutant patients had a DCR of $20 \%, 25 \%, 62 \%$, and $44 \%$, while $K R A S$ wt patients had a DCR of $36 \%, 57 \%, 49 \%$, and $47 \%$, respectively. PFS was 2.0 months for all arms and was regardless of $K R A S$ status. OS was 6.5 months, 9.0 months, 5.1 months, and 5.1 months, respectively, for $K R A S$-wt patients ( $p=0.03$ ). Further follow-up and study are clearly warranted. ${ }^{31}$ In addition, a Phase I/Ib study will evaluate pembrolizumab in combination with afatinib for EGFR-mutant NSCLC patients with acquired resistance to erlotinib in the advanced setting. This study is still early and ongoing (NCT02364609). A Phase I/Ib study will also evaluate pembrolizumab in combination with crizotinib for advanced, untreated, $A L K$-translocation NSCLC patients (NCT02364609). Pembrolizumab is also being

Table 4 Combinations with pembrolizumab

\begin{tabular}{|c|c|c|c|c|c|c|c|}
\hline Trial name & Phase & Histology & Therapy & mPFS & I-Year PFS & mORR & Ref \\
\hline \multirow[t]{3}{*}{ KEYNOTE-02I } & $\mathrm{I} / \mathrm{II}$ & All & PEMBRO+IPI & 6 months & $N R$ & $24 \%$ & 16 \\
\hline & & & & mos & I-Year OS & DOR & \\
\hline & & & PEMBRO+IPI & 17 months & NR & 14 & \\
\hline
\end{tabular}

Note: Completed trials in NSCLC.

Abbreviations: mPFS, median progression-free survival; PEMBRO, pembrolizumab; IPI, ipilimumab; mOS, median overall survival; NR, not reported; NSCLC, non-small-cell lung cancer; mORR, median overall response rate; DOR, duration of response. 
evaluated with necitumumab in advanced NSCLC, regardless of prior therapy and EGFR/ALK status (NCT02451930). These biomarker-driven studies reinforce the need for proper biomarker development to improve response in these subsets of patients.

\section{Patient selection and biomarkers of response}

Although early data suggested that patients without PDL1-positive tumor cells and surrounding T-cells may benefit from anti-PD-1 therapies, the use of this biomarker clearly selects for those patients who may benefit in the second-line setting and appears to be necessary to select those who will benefit from upfront treatment with pembrolizumab. The current evaluation of PD-L1 expression for pembrolizumab is an immunohistochemistry assay, using the PD-L1, IHC 22C3 assay from Dako North America. Scoring is based upon a tumor proportion score (TPS) according to the percentage of viable tumor cells on sample slides that expressed PD-L1. Nivolumab utilized Dako's IHC 28-8 assay and atezolizumab utilized Ventana's SP142 assay; however, Ventana's SP263 assay and E1L3N assays have also been evaluated as possible biomarker assays for PD-L1, leaving standardization of PD-L1 biomarkers unresolved. ${ }^{32}$ With the approval of pembrolizumab in the first-line setting for patients with PD-L1 $\geq 50 \%$ tumors, a standard biomarker will be necessary and Dako's assay may likely be used in this setting. ${ }^{33}$

Support for the use of the PD-L1 biomarker in the advanced setting has come from multiple studies and metaanalyses examining this question. A meta-analysis evaluated the correlation between PD-L1 expression and outcomes with the use of PD-L1 inhibition. Twelve studies were included and showed that the HR for ORR comparing PD-L1 $>1 \%$ to PD-L $1<1 \%$ tumors was 2.18 (95\% CI 1.45-3.29; $p=0.0002$ ). ORR HR for patients with PD-L1 $>5 \%$ versus PD-L1 $<5 \%$ tumors was 2.66 (95\% CI 1.74-4.07; $p<0.00001)$, while PD-L1 $>10 \%$ versus PD-L1 $<10 \%$ tumors was $3.38(95 \%$ CI $2.23-5.13 ; p<0.00001)$ and PD-L1 $>50 \%$ versus PD-L1 $<50 \%$ tumors, was 3.99 (95\% CI 2.81-5.66; $p<0.00001)$. This trend supported the idea that treatment with PD-1 inhibition such as pembrolizumab was more beneficial with higher levels of PD-L1 expression. ${ }^{34}$ Another meta-analysis evaluated 13 studies and showed only a 13\% response rate of 915 PDL1-negative patients. HR for response rate (RR) comparing PD-L1-positive versus -negative patients was 2.08 (95\% CI $1.49-2.91 ; p<0.01)$. RR correlated with increased levels of PD-L1 expression with a Pearson's correlation of 0.43 . There was a 24-week PFS benefit with increasing PD-L1 expression with an HR of 0.79 (95\% CI 0.71-0.89). The 1-year OS also showed an increased trend with PD-L1 expression with an HR of 0.96 (95\% CI 0.87-1.06). ${ }^{35}$

In addition, a study evaluating a newer scoring method for Dako's PD-L1 assay for pembrolizumab showed an RR of $43 \%$ for PD-L1 $>50 \%$ tumors compared to $8 \%$ for PD-L1 $<50 \%$ tumors. PFS was 4.0 months versus 2.1 months, and OS was NR versus 6.1 months, respectively. This further supported the findings that pembrolizumab and checkpoint blockade have higher chance of benefit in NSCLC patients with higher PD-L1 expression. ${ }^{36}$

Although multiple IHC platforms for PD-L1 exist (SP263, SP142, E1L3N, Dako 22C3, and Dako 28-8), there is some evidence that results from one platform may correlate with those from another platform. Nivolumab was evaluated in the first-line setting but used a cutoff of $1 \%$ and $5 \%$ for PD-L1 expression with Dako 28-8, while pembrolizumab utilized the Dako IHC 22C3 assay cutoff of $>50 \%$ in the first-line setting. Ventana's SP263 assay showed a similar sensitivity and specificity compared to the E1L3N stain but was darker and provided a more interpretable signal. The SP263 assay at a $25 \%$ cutoff was similar to Dako $28-8$ at the $10 \%$ cutoff, while SP263 and Dako 22C3 showed similar staining at the $50 \%$ cutoff. These three major assays are in agreement at a rate over $90 \%$, but sensitivity and specificity vary with each assay's cutoffs. Several studies have shown that the Ventana's UltraView PD-L1 22C3 assay correlated with Dako's PD-L1 IHC for eight strongly positive, seven weakly positive, and 26 negative cases $87.8 \%$ of the time with a Pearson's correlation of $0.91(p<0.0001)$. Ventana's Optiview PD-L1 22C3 assay correlated $85.3 \%$ with Dako and had a Pearson's correlation score of $0.89(p<0.0001){ }^{36,37}$ Further studies are necessary to statistically determine which of these assays are the most sensitive and specific at their respective cutoff points. As they have been studied with different drugs in both the firstand second-line settings, further study is necessary to see whether assays can be utilized appropriately between drugs that were not approved. A major caveat to utilizing the most efficacious diagnostic assays would be that said assay may not have been approved alongside the preferred therapy. Ongoing discussions between patients' oncologists and pathologists are necessary to determine appropriate stratification between PD-L1-positive versus -negative disease.

\section{Toxicity}

The initial approval study and evaluation of pembrolizumab in NSCLC with KEYNOTE-001 showed TrAEs in $70.9 \%$ or 351 of 495 of all treated patients. The most common TrAEs 
were decreased appetite, pruritus, and fatigue. Grade 3 or higher TrAEs were reported in 47 patients out of 495 patients or $9.5 \%$. Significant TrAEs included infusion reactions at $3.0 \%$, hypothyroidism in $6.9 \%$, and pneumonitis in $3.6 \%$. Grade 3 or higher pneumonitis occurred in $1.8 \%$ of patients including one patient who died. ${ }^{10}$ Similar TrAEs were also seen in the first-line study of KEYNOTE-024. TrAEs occurred in $73.4 \%$ of patients treated with pembrolizumab versus $90.0 \%$ of patients treated with chemotherapy. Grade 3 and higher TrAEs occurred in only $26.6 \%$ of patients in the pembrolizumab arm versus $53.5 \%$ of patients in the chemotherapy arm. Severe TrAEs occurred at a similar rate between both arms, with $21.4 \%$ versus $20.7 \%$, respectively, and discontinuation occurred in only $7.1 \%$ versus $10.7 \%$, respectively. One death occurred in the pembrolizumab arm that was of unknown causes, while three patients on chemotherapy died from pulmonary sepsis, diffuse alveolar hemorrhage, and unknown causes. The most common pembrolizumab-associated TrAEs were diarrhea, fatigue, and fever at $14.3 \%, 10.4 \%$, and $10.4 \%$, respectively. Grade 3 and higher TrAEs included diarrhea at 3.9\% and pneumonitis at $2.6 \%$. The chemotherapy arm had almost double the amount of severe TrAEs, including complications of cytopenias. Grade 3 and higher immune-mediated AEs occurred in $9.7 \%$ of patients with pembrolizumab versus $0.7 \%$ of patients with chemotherapy. ${ }^{14}$

Similar TrAEs have been seen across many studies for pembrolizumab in NSCLC and for checkpoint blockade in general across multiple tumor types. The most alarming AEs seen, which have been associated with rare grade 5 toxicity, include pneumonitis and colitis. Both are monitored very closely in all patients on PD-1 blockade. However, some findings are specific to particular checkpoint signals, tumor types, and even drug agents. Pembrolizumab also has some unique case-reported toxicities. A 73-year-old woman with ipilimumab-refractory, metastatic melanoma was treated with pembrolizumab and developed new-onset heart failure that was later shown to be autoimmune myocarditis. She recovered after prompt use of corticosteroids, but such events of myocarditis with the use of checkpoint blockade have been reported, including with nivolumab therapy. ${ }^{38,39}$ Another case included a 72-year-old woman with stage IV Hodgkin's lymphoma who developed new skin nodules, hilar adenopathy, dyspnea, and left eye pain, all attributed to systemic sarcoidosis 6 months into therapy with pembrolizumab. She also improved after a course of steroids and was able to remain in complete remission at the time of publication. ${ }^{40}$ A case study reported a 62-year-old patient with malignant pleural mesothelioma (MPM) who showed development of minimal change disease (MCD) on electron microscopy of renal biopsy after the development of edema and renal failure 10 days into pembrolizumab therapy, and she quickly improved on steroids. ${ }^{41}$

The relatively early data regarding toxicity with pembrolizumab in NSCLC show clear benefit over SOC, cytotoxic chemotherapy, but keen evaluation in multiple tumor types suggests that very atypical and unintuitive AEs can be seen. It has been shown that, in the melanoma population, early immune-related findings, such as cutaneous manifestations of toxicity or skin rash, are correlated with response. A study with NSCLC patients evaluated the role of cutaneous AEs and response. Patients with macular papular eruption, pruritus, or hypopigmentation ( $42 \%$ of the study patients) all had statistically significant longer PFS compared with patients with no cutaneous AEs. ${ }^{42}$ Such findings may give information regarding response, however, much information is still needed to determine which patients will not respond and which patients will develop resistance.

\section{Mechanisms of acquired resistance}

Although patients with advanced NSCLC have higher response rates to pembrolizumab compared to cytotoxic chemotherapy, not all patients respond. To date, little is known about the determinants of response beyond the level of PD-L1 positivity and potentially the antigen burden or mutational load in the tumor. ${ }^{43}$ The patients who do respond have been shown to have durable responses. However, some of these patients who develop a durable response will later progress suggesting that acquired resistance to checkpoint blockade therapy does occur. The resistance mechanisms in patients who initially respond, and then progress, on checkpoint blockade are largely unknown, but studies that elucidate these mechanisms may allow for a deeper understanding into why some patients do not respond and lead to new therapies that ensure long durations of response for our patients. Smaller studies have utilized whole-exome sequencing (WES) in melanoma patients who responded well to PD-1 inhibition (including pembrolizumab) and then progressed. The gene analysis of these patients revealed the development of mutations in B2M, JAK1, and JAK2 associated with the interferon pathway. Although this study was limited by the number of samples, it provided a crucial insight into possible immune escape mechanisms the tumor microenvironment may develop. ${ }^{44}$ Further studies on both mechanisms of de novo and acquired resistance are clearly needed. 


\section{Other thoracic cancers}

With the significant efficacy seen in NSCLC, the utility of pembrolizumab in other thoracic malignancies is being evaluated. KEYNOTE-028 is an ongoing Phase Ib study evaluating pembrolizumab in patients with advanced, PD-L1+, pretreated, small-cell lung cancer (SCLC). Patients will receive pembrolizumab $10 \mathrm{mg} / \mathrm{kg}$ every 2 weeks up to 2 years and primary end points will include response and safety. The initial 16 patients accrued showed 53\% TrAEs, only one grade 3 TrAE, four partial responses, and one patient with stable disease and six patients with progression of disease and five patients awaiting response evaluation at interim analysis. The responses observed in these patients appear to be durable and have been reported to be as high as $16+$ weeks. ${ }^{45}$ Pembrolizumab is also being evaluated in PD-L1-positive MPM in the Phase Ib setting along with other advanced, solid tumors in the KEYNOTE-028 study. Of the 25 patients accrued at the time of interim analysis, seven patients had a partial response and 12 patients had stable disease while the remaining patients were still being evaluated. ${ }^{46}$ In addition, the role of pembrolizumab in NSCLC with untreated central nervous system (CNS) metastases is also under clinical evaluation. Early data of Phase II, nonrandomized, open-label study of pembrolizumab in melanoma and NSCLC with untreated brain metastases showed activity and acceptable safety. Of the 18 patients enrolled with NSCLC with at least one untreated brain metastasis measuring 0.5 to $2.0 \mathrm{~cm}$, six patients $(33 \%$, 95\% CI 14-59\%) had a response. Four of these six patients had a complete response, and five of the six patients have ongoing responses (3.2 to 7.0 months).

\section{Conclusion}

With the advent of checkpoint blockade immunotherapy agents, such as pembrolizumab, lung cancer therapy has been drastically altered. The initial second-line approval almost doubled the response rates previously seen with SOC chemotherapy with durable responses providing meaningful time to patients, with less toxicity. The findings of KEYNOTE-024 showed the benefit of pembrolizumab over chemotherapy in the first-line setting as well for those patients with PD-L1 $\geq 50 \%$ tumors. However, many trials evaluating combinations with chemotherapy are still ongoing and awaiting analysis. Novel combinations including pembrolizumab with concurrent chemoradiation, histone deacetylase inhibitors, hypomethylating agents, TKIs such as erlotinib, PI3K inhibitors, MEK inhibitors such as selumetinib, and multiplatform adaptive studies such as BATTLE-2 are awaiting further analysis but show promise. The challenge remains to find combinations that will allow for increased and durable responses in the majority of patients who currently do not benefit from monotherapy as well as those patients where response rates are extremely low (never smokers, EGFR mutant/ALK-positive tumors).

Pembrolizumab, along with its companion diagnostic from Dako, has set the stage for checkpoint blockade. Although standardization of PD-L1 assays has not been established, pembrolizumab and Dako have shifted the paradigm for companion diagnostics, given the many biomarker-driven studies with pembrolizumab and the clear benefit of therapy in PD-L1 patients as established by the Dako IHC 22C3 assay. Even the scoring system is being replicated by other companies, with the Dako assay for pembrolizumab as the gold standard. The need for cross-validation of these assays is increasingly important as the FDA approved pembrolizumab in the first-line setting without specifying a specific PD-L1 assay to determine eligibility.

Toxicity of pembrolizumab is similar to most checkpoint blockade therapy, including autoimmune findings of rash, colitis, pneumonitis, and thyroiditis, while hypophysitis is much rarer. Rarer, but potentially serious, autoimmune side effects have been observed, as well including myocarditis, sarcoidosis, or autoimmune arthritis. Reassuringly, most patients respond to steroid therapy without developing immediate progression of disease. To date, there are little data beyond a past medical history of autoimmune disease to predict which patients will develop these toxicities. This is clearly an area that needs to be explored further to improve the safety of these agents. Other tumor types, including PD-L1+ SCLC and MPM, are also under evaluation with pembrolizumab, and early analyses are showing promise.

However, many questions regarding pembrolizumab therapy in lung cancer remain unanswered. It is unclear whether pembrolizumab is a superior PD-1 agent to nivolumab. Pembrolizumab has shown efficacy and has been approved in the first-line setting. However, the nivolumab first-line trial had a lower PD-L1 cutoff ( $>5 \%$ positivity) and with a less PD-L1 enriched population, did not meet its endpoint. Nivolumab was the first anti-PD-1 to be approved in the pretreated population, and no head-to-head comparison between the two products has been conducted. Therefore, the choice between the two agents in the pretreated setting is unclear. In addition, it is unknown whether the PD-L1 antagonists such as atezolizumab will prove to be more or less efficacious or safer than pembrolizumab. The role of pembrolizumab in the neoadjuvant and adjuvant settings is under continued evaluation along with duration of therapy. The studies in melanoma have shown ongoing responses 
with pembrolizumab out to 5 years to date, and responses can be observed with as little as one dose and so the duration of therapy is not fully established. ${ }^{48}$ Further study is clearly required to determine the optimal length of treatment with these agents to both minimize cost and toxicity. The initial approval studies showed benefit for subgroups with smoking history, which was theorized to be from an elevated mutation burden and increased tumor immunogenicity. Furthermore, studies have demonstrated minimal response rates in never smoking patients with EGFR mutations or $A L K$ translocations. ${ }^{49}$ Therefore, the role of pembrolizumab in the never smoker population is less clear. Ultimately, the data for pembrolizumab combinations, resistance mechanisms, gene expression of responders, and role in NSCLC are still nascent, but very promising.

\section{Disclosure}

TFB has served on an ad hoc steering committee for Regeneron Pharmaceuticals, Inc. The authors report no other conflicts of interest in this work.

\section{References}

1. Siegel RL, Miller KD, Jemal A. Cancer statistics, 2016. CA Cancer J Clin. 2016;66(1):7-30.

2. Hanahan D, Weinberg RA. Hallmarks of cancer: the next generation. Cell. 2011;144(5):646-674.

3. Pardoll DM. The blockade of immune checkpoints in cancer immunotherapy. Nat Rev Cancer. 2012;12(4):252-264.

4. Topalian SL, Hodi FS, Brahmer JR, et al. Safety, activity, and immune correlates of anti-PD-1 antibody in cancer. $N$ Engl J Med. 2012;366(26):2443-2454.

5. Lynch TJ, Bondarenko I, Luft A, et al. Ipilimumab in combination with paclitaxel and carboplatin as first-line treatment in stage IIIB/IV non-small-cell lung cancer: results from a randomized, double-blind, multicenter phase II study. J Clin Oncol. 2012;30(17):2046-2054.

6. Konishi J, Yamazaki K, Azuma M, Kinoshita I, Dosaka-Akita H, Nishimura M. B7-H1 expression on non-small cell lung cancer cells and its relationship with tumor-infiltrating lymphocytes and their PD-1 expression. Clin Cancer Res. 2004;10(15):5094-5100.

7. Hamid O, Robert C, Daud A, et al. Safety and tumor responses with lambrolizumab (anti-PD-1) in melanoma. N Engl J Med. 2013;369(2): 134-144.

8. Borghaei H, Paz-Ares L, Horn L, et al. Nivolumab versus docetaxel in advanced nonsquamous non-small-cell lung cancer. $N$ Engl J Med. 2015;373(17):1627-1639.

9. Brahmer J, Reckamp KL, Baas P, et al. Nivolumab versus docetaxel in advanced squamous-cell non-small-cell lung cancer. $N$ Engl J Med. 2015;373(2):123-135.

10. Garon EB, Rizvi NA, Hui R, et al; KEYNOTE-001 Investigators. Pembrolizumab for the treatment of non-small-cell lung cancer. $N$ Engl J Med. 2015;372(21):2018-2028.

11. Chatterjee M, Turner DC, Felip E, et al. Systematic evaluation of pembrolizumab dosing in patients with advanced non-small-cell lung cancer. Ann Oncol. 2016;27(7):1291-1298.

12. Merck Sharp \& Dohme Corp. Study of pembrolizumab (MK-3475) in participants with advanced non-small cell lung cancer (MK-3475025/KEYNOTE-025). Available from: https://clinicaltrials.gov/ct2/ show/NCT02007070. NLM identifier: NCT02007070. Accessed December 22, 2016.
13. Herbst RS, Baas P, Perez-Gracia JL, et al. PD1.06 (also presented as P2.41): pembrolizumab vs docetaxel for previously treated NSCLC (KEYNOTE-010): archival vs new tumor samples for PD-L1 assessment. J Thorac Oncol. 2016;11(10s):S174-S175.

14. Reck M, Rodríguez-Abreu D, Robinson AG, et al. Pembrolizumab versus chemotherapy for PD-L1-positive non-small-cell lung cancer. N Engl J Med. 2016;375(19):1823-1833.

15. Mok T, Wu YL, Sadowski S, et al. 195TiP: pembrolizumab (MK-3475) versus platinum-based chemotherapy for PD-L1+ NSCLC in a phase 3, randomized, open-label study: KEYNOTE-042. J Thorac Oncol. 2016;11(4 Suppl):S142.

16. Gadgeel SM, Stevenson J, Langer CJ, et al. Pembrolizumab (pembro) plus chemotherapy as front-line therapy for advanced NSCLC: KEYNOTE-021 cohorts A-C. J Clin Oncol. 2016;34(15 Suppl):9016.

17. Merck Sharp \& Dohme Corp. Study of pembrolizumab (MK-3475) monotherapy in advanced solid tumors and pembrolizumab combination therapy in advanced non-small cell lung cancer (MK-3475011/KEYNOTE-011). Available from: https:/clinicaltrials.gov/ct2/ show/NCT01840579. NLM identifier: NCT01840579. Accessed December 22, 2016.

18. Merck Sharp \& Dohme Corp. A study of carboplatin-paclitaxel/nabpaclitaxel chemotherapy with or without pembrolizumab (MK-3475) in adults with first line metastatic squamous non-small cell lung cancer (MK-3475-407/KEYNOTE-407). Available from: https://clinicaltrials.gov/ct2/show/NCT02775435. NLM identifier: NCT02775435. Accessed December 22, 2016.

19. Hall RD, Gadgeel SM, Garon EB, et al. Phase 3 study of platinumbased chemotherapy with or without pembrolizumab for first-line metastatic, nonsquamous non-small cell lung carcinoma (NSCLC): KEYNOTE-189. J Clin Oncol. 2016;34(15 Suppl):TS9104.

20. Merck Sharp \& Dohme Corp. Study of platinum+pemetrexed chemotherapy with or without pembrolizumab (MK-3475) in participants with first line metastatic non-squamous non-small cell lung cancer (MK3475-189/KEYNOTE-189). Available from: https://clinicaltrials.gov/ ct2/show/NCT02578680. NLM identifier: NCT02578680. Accessed December 22, 2016.

21. Merck Sharp \& Dohme Corp. Study of pembrolizumab (MK-3475) vs placebo for participants with non-small cell lung cancer after resection with or without standard adjuvant therapy (MK-3475-091/ KEYNOTE-091) (PEARLS). Available from: https://clinicaltrials.gov/ ct2/show/NCT02504372. NLM identifier: NCT02504372. Accessed December 22, 2016.

22. O'Brien MER, Hasan B, Dafni U, et al. EORTC-ETOP randomized, phase 3 trial with anti-PD-1 monoclonal antibody pembrolizumab versus placebo for patients with early stage non-small cell lung cancer (NSCLC) after resection and standard adjuvant chemotherapy: PEARLS (NCT02504372). J Clin Oncol. 2016;34(15 Suppl):TS8571.

23. Postow MA, Callahan MK, Barker CA, et al. Immunologic correlates of the abscopal effect in a patient with melanoma. N Engl J Med. 2012; 366(10):925-931.

24. Durm GA, Kio EA, Fisher WB, et al. Phase II trial of consolidation Pembrolizumab following concurrent chemoradiation in patients (pts) with unresectable or inoperable stage III non-small cell lung cancer (NSCLC): initial safety data from HCRN LUN 14-179. J Clin Oncol. 2016;34(15 Suppl):e20025.

25. Levy BP, Giaccone G, Besse B, et al. A phase II multicenter, randomized, placebo-controlled, double-blind study of CC-486 plus pembrolizumab (pembro) vs pembro plus placebo (PBO) in previously treated patients (pts) with locally advanced/metastatic non-small cell lung cancer (NSCLC). J Clin Oncol. 2016;34(15 Suppl):TS9107.

26. Ruiz R, Raez LE, Rolfo C. Entinostat (SNDX-275) for the treatment of non-small cell lung cancer. Expert Opin Investig Drugs. 2015; 24(8):1101-1109.

27. Johnson ML, Adjei AA, Ramalingam SS, et al. Preliminary results of ENCORE 601, a phase 1b/2, open-label study of entinostat (ENT) in combination with pembrolizumab (PEMBRO) in patients with nonsmall cell lung cancer (NSCLC). J Clin Oncol. 2016;34(15 Suppl): e20659. 
28. Juergens RA, Wrangle J, Vendetti FP, et al. Combination epigenetic therapy has efficacy in patients with refractory advanced non-small cell lung cancer. Cancer Discov. 2011;1(7):598-607.

29. Tolcher AW, Hong DS, Sullivan RJ, et al. IPI-549-01-A phase 1/1b first in human study of IPI-549, a PI3K-\{gamma $\}$ inhibitor, as monotherapy and in combination with pembrolizumab in subjects with advanced solid tumors. J Clin Oncol. 2016;34(15 Suppl):TS3111.

30. Gubens MA, Sequist LV, Stevenson J, et al. Phase I/II study of pembrolizumab (pembro) plus ipilimumab (ipi) as second-line therapy for NSCLC: KEYNOTE-021 cohorts D and H. J Clin Oncol. 2016;34(15 Suppl):9027.

31. Papadimitrakopoulou V, Lee JJ, Wistuba II, et al. The BATTLE-2 study: a biomarker-integrated targeted therapy study in previously treated patients with advanced non-small-cell lung cancer. J Clin Oncol. Epub 2016 Aug 1.

32. Teixido C, Karachaliou N, Gonzalez-Cao M, Morales-Espinosa D, Rosell R. Assays for predicting and monitoring responses to lung cancer immunotherapy. Cancer Biol Med. 2015;12(2):87-95.

33. Cagle PT, Bernicker EH. Challenges to biomarker testing for PD-1/ PD-L1 checkpoint inhibitors for lung cancer. Arch Pathol Lab Med. 2015;139(12):1477-1478.

34. Abdel-Rahman O. Correlation between PD-L1 expression and outcome of NSCLC patients treated with anti-PD-1/PD-L1 agents: a metaanalysis. Crit Rev Oncol Hematol. 2016;101:75-85.

35. Aguiar PN Jr, Santoro IL, Tadokoro H, et al. The role of PD-L1 expression as a predictive biomarker in advanced non-small-cell lung cancer: a network meta-analysis. Immunotherapy. 2016;8(4):479-488.

36. Roach C, Zhang N, Corigliano E, et al. Development of a companion diagnostic PD-L1 immunohistochemistry assay for pembrolizumab therapy in non-small-cell lung cancer. Appl Immunohistochem $\mathrm{Mol}$ Morphol. 2016;24(6):392-397.

37. Neuman T, London M, Kania-Almog J, et al. A harmonization study for the use of 22C3 PD-L1 immunohistochemical staining on Ventana's platform. J Thorac Oncol. 2016;11(11):1863-1868.

38. Laubli H, Balmelli C, Bossard M, Pfister O, Glatz K, Zippelius A. Acute heart failure due to autoimmune myocarditis under pembrolizumab treatment for metastatic melanoma. J Immunother Cancer. 2015;3:11.
39. Tadokoro T, Keshino E, Makiyama A, et al. Acute lymphocytic myocarditis with anti-PD-1 antibody nivolumab. Circ Heart Fail. 2016;9(10):e003514.

40. Cotliar J, Querfeld C, Boswell WJ, Raja N, Raz D, Chen R. Pembrolizumab-associated sarcoidosis. JAAD Case Rep. 2016;2(4):290-293.

41. Bickel A, Koneth I, Enzler-Tschudy A, Neuweiler J, Flatz L, Fruh M. Pembrolizumab-associated minimal change disease in a patient with malignant pleural mesothelioma. BMC Cancer. 2016;16:656.

42. Sanlorenzo M, Vujic I, Daud A, et al. Pembrolizumab cutaneous adverse events and their association with disease progression. JAMA Dermatol. 2015;151(11):1206-1212.

43. Rizvi NA, Hellmann MD, Snyder A, et al. Cancer immunology. Mutational landscape determines sensitivity to PD-1 blockade in non-small cell lung cancer. Science. 2015;348(6230):124-128.

44. Zaretsky JM, Garcia-Diaz A, Shin DS, et al. Mutations associated with acquired resistance to PD-1 blockade in melanoma. $N$ Engl $\mathrm{J} \mathrm{Med}$. 2016;375(9):819-829.

45. Ott PA, Fernandez MEE, Hiret S, et al. Pembrolizumab (MK-3475) in patients (pts) with extensive-stage small cell lung cancer (SCLC): preliminary safety and efficacy results from KEYNOTE-028. Paper presented at: ASCO Annual Meeting Proceedings; 2015.

46. Alley EW, Molife LR, Santoro A, et al. Abstract CT103: clinical safety and efficacy of pembrolizumab (MK-3475) in patients with malignant pleural mesothelioma: preliminary results from KEYNOTE-028. Cancer Res. 2015;75(15 Suppl):CT103-CT103.

47. Goldberg SB, Gettinger SN, Mahajan A, et al. Pembrolizumab for patients with melanoma or non-small-cell lung cancer and untreated brain metastases: early analysis of a non-randomised, open-label, phase 2 trial. Lancet Oncol. 2016;17(7):976-983.

48. Davar D, Socinski MA, Dacic S, Burns TF. Near complete response after single dose of nivolumab in patient with advanced heavily pretreated KRAS mutant pulmonary adenocarcinoma. Exp Hematol Oncol. 2015;4:34

49. Rizvi NA, Rusch V, Pao W, et al. Molecular characteristics predict clinical outcomes: prospective trial correlating response to the EGFR tyrosine kinase inhibitor gefitinib with the presence of sensitizing mutations in the tyrosine binding domain of the EGFR gene. Clin Cancer Res. 2011;17(10):3500-3506.
Lung Cancer: Targets and Therapy

\section{Publish your work in this journal}

Lung Cancer: Targets and Therapy is an international, peer-reviewed, open access journal focusing on lung cancer research, identification of therapeutic targets and the optimal use of preventative and integrated treatment interventions to achieve improved outcomes, enhanced survival and quality of life for the cancer patient. Specific topics covered in the journal include: Epidemiology, detection and screening Cellular research and biomarkers; Identification of biotargets and agents with novel

Submit your manuscript here: https://www.dovepress.com/lung-cancer-targets--therapy-journa
Dovepress

mechanisms of action; Optimal clinical use of existing anticancer agents, including combination therapies; Radiation and surgery; Palliative care; Patient adherence, quality of life, satisfaction; Health economic evaluations. The manuscript management system is completely online and includes a very quick and fair peer-review system. Visit http://www.dovepress.com/testimonials.php to read real quotes from published authors. 